\title{
Spatial Decay Analysis in Interconnected Dynamical Systems Using Vector Lyapunov Functions
}

\author{
Frederik Deroo*, Sandra Hirche, Brian D. O. Anderson
}

\begin{abstract}
In this paper we analyze the propagation of input signals in a large-scale network of dynamical systems. Using vector Lyapunov functions, the individual multidimensional subsystems are first reduced to an approximating scalar representation in the form of the evolution of their weighted norm. The norm simultaneously qualifies as a local Lyapunov function for the isolated subsystem. Employing properties of Mmatrices, we then derive linear system dynamics which provide an upper bound for the evolution of the original system, and use them to investigate the decay between hops from subsystem to subsystem of the steady state magnitude. Two input signals are considered: Constant input, and a sinusoidal input. The results are demonstrated using numerical simulations.
\end{abstract}

\section{INTRODUCTION}

The analysis of large-scale interconnected systems has recently become a focus of research in the field of system and control theory with the goal of solving new technological challenges in terms of ever-increasing system sizes and stricter performance requirements. This system class encompasses a large number of practical applications, including traffic systems, vehicle formations, the power system and other types of distribution and infrastructure networks, just to name a few.

One important question of interest for interconnected systems which we address in this paper is under which conditions a disturbance at one node will have little effect at another node that is far away in terms of the number of hops between the nodes. The investigation of this question is relevant for several reasons. First, it will help in understanding how disturbances, or changes in system dynamics at one subsystem, affect the behavior at distant nodes. In other words, it is of interest how the performance of a distant node is affected by local disturbances. This will lead to an intuition regarding how networks should be designed, e.g. with regards to disturbance attenuation, since it will clearly be preferred in most applications that local changes in dynamics should not have a large effect at large distances. Second, a property of this type would allow the agents more freedom even though they are connected, and since privacy is becoming an important issue, subsystems may not want all other participants in an interconnected system to be able to sense their state. Last, it would help in the development or modification of a local control design at one agent with

*Corresponding author.

F. Deroo and S. Hirche are with the Chair of Information-Oriented Control, Technische Universität München, D-80333 München, Germany, fred.deroodtum.de, hircheatum.de

B.D.O. Anderson is with The Australian National University and National ICT Australia, Canberra ACT 2600 Australia. brian.andersoneanu.edu.au limited model information from the overall system, see e.g. [1], and may enable local control law adaptations without significantly sacrificing overall system performance.

Even though this problem is practically relevant and far from trivial, the authors are not aware of many previous results in the literature that treat it directly. However, the decay property for constant disturbances turns out to be related to a decay property of entries of the inverse of a matrix, whose structure captures that of the underlying system graph, with a path graph corresponding to a tridiagonal matrix. In [2], for a symmetric tridiagonal matrix, a decay property along the rows and columns of the inverse is developed. In this paper a similar result is derived but for much more general matrix structures. Also related are the results in [3] where the authors investigate properties of M-matrices in the context of linear systems of equations, and the sensitivity of the solution to changes in the entries of the M-matrix. For the special case of directed lattices with leader-follower-dynamics, the authors in [4] derive transfer functions from disturbances to the states of nodes. This goes in a similar direction as this paper, however we consider more general dynamics and graphs. System analysis of positive systems, the type of dynamics that is eventually considered here, is treated in [5]. Also, a similar research direction is taken in [6] where a local average consensus algorithm is developed which involves both temporal and decaying spatial behavior. Our goal is to take that viewpoint of considering both temporal and spatial behavior as well, and investigate how physical signals propagate spatially through a dynamical system and if the gain over a multihop path can be bounded in terms of the hop count.

The main contribution of this paper is an analytical investigation of the decay between subsystems in response to a steady state disturbance. We consider the case of a constant input at a single node, and then look at the generalization to a sinusoidal input. It is shown that the value of the steady state response at a node can be bounded by a linear combination with positive weights of steady state values at nodes closer to the source node, and that the coefficients in that combination sum to less than one. The results are illustrated using numerical simulations. In order to obtain meaningful results, certain assumptions on the system parameters need to be made. Therefore, in this paper we consider stable systems whose stability can be shown using a vector-Lyapunov-function. The concept and the idea of vector-Lyapunov functions is summarized well in [7]. We think this assumption is reasonable on several grounds. First, the analysis in this type of setup is known 
to remain valid even if interaction gains change, a property which makes the results more robust. Second, it allows the analysis of nonlinear large-scale systems using linear system tools. Last, it is an old and in a sense tested theory with many established results.

The remainder of the paper is organized as follows. In the next section, the considered system model is introduced and the problem is formulated. In Section III the main analytical results are presented. Section IV provides numerical demonstration and illustration of the results. Last, a concluding remark is given in Section V.

Notation: We shall use the notation $X \succ 0$ to denote that $X$ is a positive definite matrix, for vectors $d \in \mathbb{R}^{N}$, $d>0$ denotes positivity of all entries. Furthermore, the notation $X=\left(X_{i j}\right)$ means that the matrix $X$ is made up of several matrix blocks $X_{i j}$. The minimum and maximum eigenvalue of a matrix $X$ are denoted by $\lambda_{m}(X)$ and $\lambda_{M}(X)$, the maximum singular value is $\sigma_{M}(X)$.

\section{SYSTEM DESCRIPTION AND PROBLEM FORMULATION}

In this section, we introduce the considered problem, and review the concept at the core of this work, namely vector Lyapunov functions.

We begin with an important Lemma.

Lemma 1: [8, §6.2] A matrix $M$ is a non-singular $M$ matrix if it has positive diagonal elements, nonpositive offdiagonal elements and if it satisfies the following equivalent conditions:

- There exists a vector $d>0$ such that $M d>0$.

- There exists a positive diagonal matrix $D=$ $\operatorname{diag}\left(d_{1}, \ldots, d_{N}\right)$ such that $M D$ is strictly diagonally dominant, i.e. $d_{i} M_{i i}>\sum_{j \neq i} d_{j}\left|M_{i j}\right| \forall i=1, \ldots, N$.

In this paper, we consider an interconnected large-scale linear system which consists of $N$ subsystems. The dynamics of the $i$ th subsystem, including any local controller, are given by

$$
\dot{x}_{i}=A_{i i} x_{i}+\sum_{\substack{j=1 \\ j \neq i}}^{N} A_{i j} x_{j},
$$

where $x_{i} \in \mathbb{R}^{n_{i}}$, and $A_{i j} \in \mathbb{R}^{n_{i} \times n_{j}}$.

The interconnection structure of the subsystems is described by a directed graph $\mathcal{G}=(\mathcal{V}, \mathcal{E})$, where the node set $\mathcal{V}$ represents the subsystems, and there is an edge $(i, j) \in \mathcal{E}$ iff $A_{i j} \neq 0$. That means that an edge $(i, j)$ in the graph means that $i$ accesses $j$ to update its state. We denote the set of neighboring nodes of node $i$, i.e. those nodes that affect it, by $\mathcal{N}_{i}=\{j \mid(i, j) \in \mathcal{E}\}$.

The overall system is a concatenation of the individual subsystems, i.e.

$$
\dot{x}=A x,
$$

where $\quad x=\left[x_{1}^{T}, \ldots, x_{N}^{T}\right]^{T} \in \mathbb{R}^{n}, \quad A=\left(A_{i j}\right) \in \mathbb{R}^{n \times n}$, and $n=\sum_{i=1}^{N} n_{i}$. We assume that the isolated, decoupled subsystems described by

$$
\dot{x}_{i}=A_{i i} x_{i}
$$

are asymptotically stable. Hence, for any $Q_{i} \succ 0$ there is a $P_{i} \succ 0$ such that

$$
P_{i} A_{i i}+A_{i i}^{T} P_{i}=-Q_{i}
$$

and then clearly

$$
\nu_{i}=\left(x_{i}^{T} P_{i} x_{i}\right)^{\frac{1}{2}}
$$

is a local Lyapunov function for the isolated subsystem $i$, where with (4) we have that $\dot{\nu}_{i, \text { iso }}=-\frac{1}{2}\left(x_{i}^{T} P_{i} x_{i}\right)^{-\frac{1}{2}} x_{i}^{T} Q_{i} x_{i}<0$ is the isolated derivative of $\nu_{i}$ along the dynamics (3).

With the following bounds

$$
\begin{aligned}
\sqrt{\lambda_{m}\left(P_{i}\right)}\left\|x_{i}\right\| & \leq \nu_{i}\left(x_{i}\right) \leq \sqrt{\lambda_{M}\left(P_{i}\right)}\left\|x_{i}\right\| \\
\left\|\frac{\partial \nu_{i}}{\partial x_{i}}\right\| & =\left\|\left(x_{i}^{T} P_{i} x_{i}\right)^{-\frac{1}{2}} P_{i} x_{i}\right\| \leq \frac{\lambda_{M}\left(P_{i}\right)}{\sqrt{\lambda_{m}\left(P_{i}\right)}} \\
\left\|A_{i j} x_{j}\right\| & \leq \sigma_{M}\left(A_{i j}\right)\left\|x_{j}\right\|
\end{aligned}
$$

we can write the coupled derivative of $\nu_{i}$ as

$$
\begin{aligned}
\dot{\nu}_{i} & =\dot{\nu}_{i, \text { iso }}+\left(x_{i}^{T} P_{i} x_{i}\right)^{-\frac{1}{2}} x_{i}^{T} P_{i} \sum_{\substack{j=1 \\
j \neq i}}^{N} A_{i j} x_{j} \\
& \leq-\frac{\lambda_{m}\left(Q_{i}\right)}{2 \sqrt{\lambda_{M}\left(P_{i}\right)}}\left\|x_{i}\right\|+\sum_{\substack{j=1 \\
j \neq i}}^{N} \frac{\lambda_{M}\left(P_{i}\right) \sigma_{M}\left(A_{i j}\right)}{\sqrt{\lambda_{m}\left(P_{i}\right)}}\left\|x_{j}\right\| \\
& \leq-\tilde{\alpha}_{i, i} \nu_{i}+\sum_{\substack{j=1 \\
j \neq i}}^{N} \tilde{\alpha}_{i, j} \nu_{j},
\end{aligned}
$$

where $\tilde{\alpha}_{i, i}=\frac{\lambda_{m}\left(Q_{i}\right)}{2 \lambda_{M}\left(P_{i}\right)}, \quad \tilde{\alpha}_{i, j}=\frac{\lambda_{M}\left(P_{i}\right) \sigma_{M}\left(A_{i j}\right)}{\sqrt{\lambda_{m}\left(P_{i}\right)} \sqrt{\lambda_{m}\left(P_{j}\right)}}$.

Theorem 1: [7], [9] Consider system (2), and the local Lyapunov functions (5) with $P_{i}$ and $Q_{i i}$ from (4). If the matrix $-\tilde{\mathcal{A}}$ with elements from (7)

$$
\tilde{\mathcal{A}}_{i j}= \begin{cases}-\tilde{\alpha}_{i, i} & \text { if } i=j \\ \tilde{\alpha}_{i, j} & \text { if } i \neq j\end{cases}
$$

is a non-singular M-matrix, then the system (2) is stable and $V(x)=d^{T} \nu(x)$, where $d=\left[d_{1}, \ldots, d_{N}\right]^{T}>0$ and $\nu=\left[\nu_{1}\left(x_{1}\right), \ldots, \nu_{N}\left(x_{N}\right)\right]^{T}$ is a Lyapunov function for the system. Furthermore, we call $\nu$ with $\dot{\nu} \leq \tilde{\mathcal{A}} \nu$ a vector Lyapunov function of system (2).

There are several other ways to set up a vector Lyapunov function to show stability but since stability investigations are not the focus of this paper, we refer the reader to $[7, \S 2]$ for more details. ${ }^{1}$

In the following, we use the dynamics of $\nu_{i}$ to investigate signal decay in the interconnected system. By the comparison principle $[9, \S 1.4]$, instead of the inequality (7) we

\footnotetext{
${ }^{1}$ Note that due to the use of vector Lyapunov function theory, the results in this paper are extendable to a wide class of nonlinear systems. Necessary assumptions are the existence of constants $c_{1, i}>0, c_{2, i}>0$ such that $c_{1, i}\left\|x_{i}\right\| \leq \nu_{i} \leq c_{2, i}\left\|x_{i}\right\|$, and the interconnection between subsystems needs to be bounded. For ease of exposition we restrict our attention in this paper to linear systems.
} 
consider $\tilde{w}(t)$ with $\tilde{w}(0)=\nu(0)$ and

$$
\begin{aligned}
\dot{\tilde{w}}_{i} & =-\tilde{\alpha}_{i, i} \tilde{w}_{i}+\sum_{\substack{j=1 \\
j \neq i}}^{N} \tilde{\alpha}_{i, j} \tilde{w}_{j}, \\
\text { or } \quad \dot{\tilde{w}} & =\tilde{\mathcal{A}} \tilde{w},
\end{aligned}
$$

where $\tilde{w} \in \mathbb{R}^{N}$ and $\tilde{\mathcal{A}} \in \mathbb{R}^{N \times N}$. It is clear that $\nu_{i}(t) \leq \tilde{w}_{i}(t)$. That means that the system (8) in a limited and special way approximates system (1) as a scalar system, and we can consider $\tilde{w}(t)$ as a super-state for the actual system state $x(t)$. In particular, we will use it to make estimates on the signal decay in interconnected systems. As already mentioned in Section I, we make the following assumption on system (9).

Assumption 1: $-\tilde{\mathcal{A}}$ is a non-singular M-matrix.

Then there is a positive diagonal matrix $D$ such that the matrix $\mathcal{A}:=D^{-1} \tilde{\mathcal{A}} D$ with elements $\alpha_{i j}$ is row diagonally dominant [8], i.e.

$$
\alpha_{i, i}-\sum_{j \in \mathcal{N}_{i}} \alpha_{i, j} \geq 0 \forall i .
$$

This also implies that the following condition holds

$$
\alpha_{i, i}-\frac{\sum_{j \in \mathcal{N}_{i}} \alpha_{i, j}}{1-\gamma} \geq 0 \forall i,
$$

for a known $0<\gamma<1$, where equality holds for at least one $i$.

Hence, $\gamma$ can be seen as a measure of how row dominant the matrix $\mathcal{A}$ is. If the original system does not lead to satisfaction of Assumption 1, the authors in [10] present a system class for which decentralized controllers can be designed such that Assumption 1 is satisfied. The identification of further system classes where distributed controllers lead to Assumption 1 being satisfied is also current work. As a side note: Using (10) and Gershgorin arguments, the eigenvalues of $\mathcal{A}$ can easily be bounded.

\section{ANALYSIS}

In this section, we present the main analytical results, namely the investigation of a way to describe the steady state of system (9) for two cases: 1) The system has a scalar constant input at a single node. 2) The system has a scalar sinusoidal input at a single node. That way we want to develop an insight as to how a physical signal propagates spatially from system to system.

\section{A. Steady state decay with constant input}

In this subsection we investigate how the steady state of an interconnected system behaves when there is one node with a constant and positive external input and the remaining nodes are not excited. The system dynamics (9) are transformed according to Assumption 1 and then extended to include the input and we obtain

$$
\dot{w}=\mathcal{A} w+b u,
$$

where $b \in \mathbb{R}^{N}$ is a vector consisting of only one nonzero entry, namely a one, and for convenience of derivation and presentation $u>0 \in \mathbb{R}$. These are the system dynamics that will be considered for the rest of the paper. Notice that the graphical structure of $\mathcal{A}$ is identical to the graph $\mathcal{G}$, i.e. there is an edge from node $i$ to node $j$ when $i$ uses information from $j$ to update its state, in other words when $i$ is influenced by $j$.

Remark 1: Since the setup is linear, by the superposition principle the results are straightforward to expand to multiple or negative inputs. Also since only the limit case $t \rightarrow \infty$ is considered, the results also cover inputs that become constant after a finite time.

In the following, we consider the steady state of $w$ which we denote by $\bar{w}$, i.e. $\lim _{t \rightarrow \infty} w(t)=\bar{w}$.

Definition 1: Let $\bar{w}_{i} \in \mathbb{R}, \bar{w}_{j} \in \mathbb{R}$ for $j \in\left\{j_{1}, \ldots, j_{l}\right\}$, where $i \notin\left\{j_{1}, \ldots, j_{l}\right\}$, and $0<\gamma<1$ from (10). If there exist $\beta_{i, j}$ for $j \in\left\{j_{1}, \ldots, j_{l}\right\}$ such that

$$
\bar{w}_{i}=\sum_{k=j_{1}}^{j_{l}} \beta_{i, k} \bar{w}_{k},
$$

where $\quad \sum_{k=j_{1}}^{j_{l}} \beta_{i, k}<1-\gamma \quad$ and $0 \leq \beta_{i, j}<1-\gamma \quad$ for all $j \in\left\{j_{1}, \ldots, j_{l}\right\}$, then $\bar{w}_{i}$ is termed a steady state conic combination (SSCC) of the steady states $\bar{w}_{j}$ with $j \in\left\{j_{1}, \ldots, j_{l}\right\}$. This is written as as $\bar{w}_{i} \in C\left(\bar{w}_{j_{1}}, \ldots, \bar{w}_{j_{l}}\right)$.

Note that $C(\ldots)$ is a special case of a conic combination of steady state values. With this notion of SSCC, it can be shown that if $\bar{w}_{i}$ is an SSCC of the steady states of some nodes $j_{1}, \ldots, j_{l}$, and if the steady state of one of those nodes $\bar{w}_{j_{l}}$ is an SSCC of the steady states of $i$ and other nodes $k_{1}, \ldots, k_{m}$, then $\bar{w}_{j_{l}}$ can be replaced in the SSCC of $\bar{w}_{i}$ by the steady states of nodes $k_{1}, \ldots, k_{m}$. We summarize this more formally in the following lemma.

Lemma 2:

If $\bar{w}_{i} \in C\left(\bar{w}_{j_{1}}, \ldots, \bar{w}_{j_{l}}\right)$ and $\bar{w}_{j_{l}} \in C\left(\bar{w}_{i}, \bar{w}_{k_{1}}, \ldots, \bar{w}_{k_{m}}\right)$, then $\bar{w}_{i} \in C\left(\bar{w}_{j_{1}}, \ldots, \bar{w}_{j_{l-1}}, \bar{w}_{k_{1}}, \ldots, \bar{w}_{k_{m}}\right)$ for $i \notin$ $\left\{j_{1}, \ldots, j_{l}\right\}$.

Proof:

Using (12) we start with

$$
\begin{aligned}
\bar{w}_{i} & =\beta_{i, j_{1}} \bar{w}_{j_{1}}+\ldots+\beta_{i, j_{l-1}} \bar{w}_{j_{l-1}} \\
& +\beta_{i, j_{l}}\left(\beta_{j_{l}, i} \bar{w}_{i}+\beta_{j_{l}, k_{1}} \bar{w}_{k_{1}}+\ldots+\beta_{j_{l}, k_{m}} \bar{w}_{k_{m}}\right)
\end{aligned}
$$

and obtain

$$
\begin{aligned}
\bar{w}_{i} & =\frac{\beta_{i, j_{1}}}{1-\beta_{i, j_{l}} \beta_{j_{l}, i}} \bar{w}_{j_{1}}+\ldots+\frac{\beta_{i, j_{l}-1}}{1-\beta_{i, j_{l}} \beta_{j_{l}, i}} \bar{w}_{j_{l-1}} \\
& +\frac{\beta_{i, j_{l}} \beta_{j_{l}, k_{1}}}{1-\beta_{i, j_{l}} \beta_{j_{l}, i}} \bar{w}_{k_{1}}+\ldots+\frac{\beta_{i, j_{l}} \beta_{j_{l}, k_{m}}}{1-\beta_{i, j_{l}} \beta_{j_{l}, i}} \bar{w}_{k_{m}} .
\end{aligned}
$$

Using the conditions of the lemma and (12) one can easily see that all coefficients on the right in (13) and their sum are smaller than $1-\gamma$, i.e.

$$
\begin{aligned}
\frac{1}{1-\beta_{i, j_{l}} \beta_{j_{l}, i}}\left(\sum_{r=1}^{l-1} \beta_{i, j_{r}}+\beta_{i, j_{l}} \sum_{s=1}^{m} \beta_{j_{l}, k_{s}}\right) & <1-\gamma, \\
\frac{\beta_{i, j_{r}}}{1-\beta_{i, j_{l}} \beta_{j_{l}, i}} & <1-\gamma, \quad \frac{\beta_{i, j_{l}} \beta_{j_{l}, k_{s}}}{1-\beta_{i, j_{l}} \beta_{j_{l}, i}}<1-\gamma,
\end{aligned}
$$




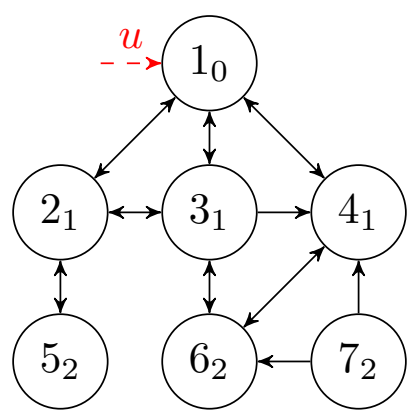

Fig. 1: Example graph. The number denotes the nodes, the index denotes the distance to the source node. Note that the input is not an edge of the graph and does not follow the edge direction convention of the rest of the graph.

for $1 \leq r \leq l-1$ and $1 \leq s \leq m$. Notice that based on Definition 1, the lemma also subsumes the case where we only have $\bar{w}_{j_{l}} \in C\left(\bar{w}_{k_{1}}, \ldots, \bar{w}_{k_{m}}\right)$ instead of $\bar{w}_{j_{l}} \in C\left(\bar{w}_{i}, \bar{w}_{k_{1}}, \ldots, \bar{w}_{k_{m}}\right)$. The reason is that this is equivalent to setting $\beta_{j_{l}, i}$ to zero, which only decreases the coefficients in the right hand side of equation (13). This concludes the proof.

Definition 2: [11] A directed path in a directed graph $\mathcal{G}$ is a sequence $v_{0}, e_{1}, v_{1}, \ldots, e_{n}, v_{r}$ such that $v_{i} \in \mathcal{V}$ and $v_{i} \neq v_{j}$ for all $0 \leq i, j \leq r$, and such that $e_{i} \in \mathcal{E}$ is a directed edge from $v_{i-1}$ to $v_{i}$ for every $1 \leq i \leq r$.

For example, in Figure 1, the node sequence $(7,4,3)$ with its corresponding edges does not constitute a directed path, but the node sequence $(7,6,3)$ does.

Definition 3: Given a designated source node in the interconnection graph $\mathcal{G}$ associated with the system dynamics (11), the distance of a node is the least number of directed edges across all paths between the node and the source node.

Definition 4: Given a designated source node in the interconnection graph $\mathcal{G}$ associated with the system dynamics (11), and given a node $v$ with distance $d$ from the source node, the unique $(d-1)$-paths are those paths starting at $v$ and ending at a node with distance $(d-1)$ without containing any other node with distance $(d-1)$. The set of nodes that can be reached via a unique $(d-1)$-path from node $v$ are called the unique $(d-1)$-nodes and is denoted by $\mathcal{V}_{v, d-1}$.

Seen from a different point of view, if there is a path from $v_{1}$ with distance $d$ to the source node, and that path has only strictly decreasing distance after reaching a node $v_{2}$ with distance $(d-1), v_{2}$ belongs to the set $\mathcal{V}_{v_{1}, d-1}$. For example, in Figure 1, we have the sets $\mathcal{V}_{6, d-1}=\{3,4\}$, $\mathcal{V}_{7, d-1}=\{3,4\}$ and $\mathcal{V}_{5, d-1}=\{2\}$.

In the following, there will be a slight abuse of notation: When $\bar{w}_{i}$ is an SSCC of the steady states of some set of nodes $\overline{\mathcal{V}}=\left\{j_{1}, \ldots, j_{l}\right\}$, instead of having to write $\bar{w}_{i} \in C\left(\bar{w}_{j_{1}}, \ldots, \bar{w}_{j_{l}}\right)$, , where $\bar{w}_{j_{k}}$ is the steady state of node $j_{k}$, we will just write $\bar{w}_{i} \in C(\overline{\mathcal{V}})$.

Theorem 2: Given the system dynamics (11), Assumption 1 with (10), and a constant input $u>0$ at a designated source node, the steady state value of a node $v$ with distance $d$ from the source node is an SSCC as defined in (12) of the steady state values of unique $(d-1)$-nodes of node $v$, i.e.

$$
\bar{w}_{v} \in C\left(\mathcal{V}_{v, d-1}\right) .
$$

Proof:

Given a designated source node, we denote the set of nodes with distance $d$ from that source node by $\mathcal{V}_{d}$. The maximum distance in the network is $d_{M}$. All the nodes on level $d_{M}$ from the source node either have only neighbors in the set $\mathcal{V}_{d_{M}-1}$ or additionally in the set $\mathcal{V}_{d_{M}}$. For any node $v_{d_{M}} \in \mathcal{V}_{d_{M}}$, because of (10) we have

$$
\bar{w}_{v_{d_{M}}} \in C\left(\mathcal{N}_{d_{M}, v_{d_{M}}}, \mathcal{N}_{d_{M}-1, v_{d_{M}}}\right)
$$

where the set $\mathcal{N}_{d_{M}, v_{d_{M}}}$ denotes the neighboring nodes of node $v_{d_{M}}$ with distance $d_{M}$ from the source node, and it could be the empty set, while $\mathcal{N}_{d_{M}, v_{d_{M}-1}}$ denotes those with distance $d_{M}-1$. In this case we have $\bar{w}_{v_{d_{M}}} \in C\left(\mathcal{N}_{d_{M}-1, v_{d_{M}}}\right)$ and the result of Theorem 2 is obvious. Otherwise, suppose node $v_{d_{M}}$ is connected to nodes $v_{1, d_{M}}, \ldots, v_{n, d_{M}}$. Then we will have

$$
\bar{w}_{v_{d_{M}}} \in C\left(\bar{w}_{v_{1, d_{M}}}, \ldots, \bar{w}_{v_{n, d_{M}}}, \mathcal{N}_{d_{M}-1, v_{d_{M}}}\right) .
$$

Applying Lemma 2 to the $\bar{w}_{v_{i, d_{M}}}$ replaces them with their neighbors, all of which again belong to the sets $\mathcal{V}_{d_{M}}$ or $\mathcal{V}_{d_{M}-1}$, and all can be reached from node $v_{d_{M}}$ via a directed path. Eventually, Lemma 2 can be used repeatedly to cancel out all nodes on the layer $d_{M}$, i.e. those belonging to the set $\mathcal{V}_{d_{M}}$, and replace them by nodes from the set $\mathcal{V}_{d_{M}-1}$. In the end $\bar{w}_{v_{d_{M}}}$ is an SSCC of nodes on the level $d_{M}-1$ that can be reached from $v_{d_{M}}$ via a directed path through its multihop neighbors on the level $d_{M}$. However, note that there may be nodes on the level $d_{M}-1$ that do not have neighbors in the part of the layer $d_{M}$ that can be reached from $v_{d_{M}}$ via several hops. Furthermore, there are nodes on the level $d_{M}-1$ that can only be reached through nodes on the level $d_{M}-1$, i.e. nodes, not belonging to $\mathcal{V}_{v_{d_{M}}, d-1}$. Both types of nodes do not occur in any of the SSCCs of the multihop neighbors and thus we obtain

$$
\begin{aligned}
\bar{w}_{v_{d_{M}}} \in & C\left(\mathcal{N}_{d_{M}-1, v_{d_{M}}}, \mathcal{N}_{d_{M}-1, v_{1, d_{M}}}, \ldots,\right. \\
& \mathcal{N}_{d_{M}-1, v_{n, d_{M}}}, \\
& \left.\mathcal{N}_{d_{M}-1, \mathcal{N}_{d_{M}, v_{1, d_{M}}}}, \ldots, \mathcal{N}_{\left.d_{M}-1, \mathcal{N}_{d_{M}, v_{n, d_{M}}}, \ldots\right)}\right)
\end{aligned}
$$

so we have the result of Theorem 2 for the nodes with maximum distance.

We proceed to the level with distance $d_{M}-1$, and choose a node of interest, call it $v_{d_{M}-1}$. On this level, each node can have neighbors on the current level, the one below and the one above, i.e.

$$
\bar{w}_{v_{d_{M}-1}} \in C\left(\mathcal{N}_{d_{M}-1, v_{d_{M}-1}}, \mathcal{N}_{d_{M}, v_{d_{M}-1}}, \mathcal{N}_{d_{M}-2, v_{d_{M}-1}}\right) .
$$

Using the results applying to nodes from level $d_{M}$ and Lemma 2, the neighboring sets of nodes from level $d_{M}$ 
can be replaced by multihop neighborhood nodes from the current level, i.e.

$$
\begin{gathered}
\bar{w}_{v_{d_{M}-1}} \in C\left(\mathcal{N}_{d_{M}-1, v_{d_{M}-1}}, \mathcal{N}_{d_{M}-1, \mathcal{N}_{d_{M}, v_{d_{M}-1}}},\right. \\
\left.\mathcal{N}_{d_{M}-2, v_{d_{M}-1}}\right) .
\end{gathered}
$$

The second term in the parentheses describes those nodes in the set $\mathcal{V}_{d_{M-1}}$ that are not direct neighbors of node $v_{d_{M}-1}$,

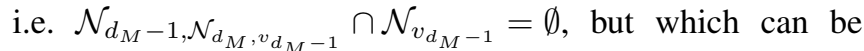
reached from $v_{d_{M}-1}$ through nodes from the set $\mathcal{V}_{d_{M}}$ via a directed path. We proceed by reasoning the same way as previously and replace all dependencies on nodes from the current level $d_{M}-1$ with nodes from the set $\mathcal{V}_{d_{M}-2}$ using Lemma 2 repeatedly. All these neighborhoods on layer $d_{M}-2$ contain nodes that can be reached either directly from $v_{d_{M}-1}$ or through other nodes on the current layer $d_{M}-1$ or the previous layer $d_{M}$. Notice again that there may be nodes with distance $d_{M}-2$ that cannot be reached from $v_{d_{M}-1}$ or any of its multihop neighbors and therefore never occur in their neighborhoods and their SSCCs. Thus they can never enter the description of $v_{d_{M}-1}$ and the result of Theorem 2 is achieved. The levels $\quad d_{M}-2 \leq d \leq 1$ can be treated in the same way which concludes the proof.

\section{Example 1: Tridiagonal systems}

For tridiagonal systems represented by a line graph as shown in Figure $2 \mathrm{a}$ it can easily be shown that the gain from one node to the next as we move away from the source node is less than $1-\gamma$. At the end of the line we have

$$
\bar{w}_{N}=\frac{\alpha_{N, N-1}}{\alpha_{N, N}} \bar{w}_{N-1}=\beta_{N, N-1} \leq(1-\gamma) \bar{w}_{N-1}
$$

and for all other nodes with $i=2, \ldots, N-1$

$$
\bar{w}_{i}=\frac{\alpha_{i, i-1}}{\alpha_{i, i}-\alpha_{i, i+1} \beta_{i+1, i}} \bar{w}_{i-1}=\beta_{i, i-1} \leq(1-\gamma) \bar{w}_{i-1}
$$

Furthermore, at the source node, one has

$$
\bar{w}_{1}=\frac{1}{\alpha_{1,1}-\alpha_{1,2} \beta_{2,1}} u .
$$

Clearly, there is a strict decay from $\bar{w}_{1}$ as we step through each one of the nodes to $\bar{w}_{N}$ and the gain is bounded by $1-\gamma$ per hop, or by $(1-\gamma)^{d}$, where $d$ is the distance of a node from the source node.

Note that this result can easily be generalized to having the input at an arbitrary node $\tilde{i}$ as depicted in Figure 2b. One

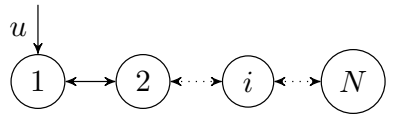

(a) Input at node 1
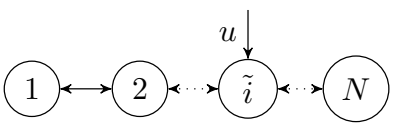

(b) Input at node $\tilde{i}$
Fig. 2: Line graph topology obtains the relationships

$$
\begin{aligned}
\bar{w}_{i} & =\frac{\alpha_{i, i+1}}{\alpha_{i, i}-\alpha_{i, i-1} \beta_{i-1, i}} \bar{w}_{i+1} \\
& =\beta_{i, i+1} \bar{w}_{i+1}<(1-\gamma) \bar{w}_{i+1}, i=1, \ldots, \tilde{i}-1, \\
\bar{w}_{i} & =\frac{\alpha_{i, i-1}}{\alpha_{i, i}-\alpha_{i, i+1} \beta_{i+1, i}} \bar{w}_{i-1} \\
& =\beta_{i, i-1} \bar{w}_{i-1}<(1-\gamma) \bar{w}_{i-1}, i=\tilde{i}+1, \ldots, N .
\end{aligned}
$$

For $i=\tilde{i}$, i.e. the node with excitation, we have

$$
\bar{w}_{\tilde{i}}=\frac{1}{\alpha_{\tilde{i}, \tilde{i}}-\alpha_{\tilde{i}, \tilde{i}+1} \beta_{i+1, i}-\alpha_{\tilde{i}, \tilde{i}-1} \beta_{i-1, i}} u .
$$

This shows that we have the same result as before, only that now the decay is in both directions, and in steady state each direction is essentially independent of the other one. Again, it is straightforward to extend this result to more general but related topologies like stars or trees.

\section{B. Steady state magnitude decay with sinusoidal input}

In this subsection we examine the same scenario as in the previous one only now instead of $u(t)=|u|$ we consider $u(t)=|u| e^{j \omega t}$, i.e. we have a sinusoidal input. While in one sense the results of the previous subsection are a special case corresponding to $\omega=0$ of the results of this subsection, we will see here that the results for general $\omega$ are best viewed in the light of those for $\omega=0$. Because the system is stable, the steady states of the subsystems will also be of sinusoidal form with the same frequency but with a constant phase shift, so we have

$$
\bar{w}_{i}(t)=\left|\bar{w}_{i}\right| e^{j\left(\omega t+\varphi_{i}\right)}
$$

for $t$ after an initial settling time, and we define the vector $\varphi=\left[\varphi_{1}, \ldots, \varphi_{N}\right]$.

For a start, we only consider the line graph case. Starting at the end, only considering the magnitudes and by using the differential equation (8) as well as the assumed solutions (18) we get the relationship

$$
\begin{aligned}
\left|\bar{w}_{N}\right| & =\frac{\alpha_{N, N-1}}{\sqrt{\alpha_{N, N}^{2}+\omega^{2}}}\left|\bar{w}_{N-1}\right|=\beta_{N, N-1}(\omega, \varphi)\left|\bar{w}_{N-1}\right| \\
& <(1-\gamma)\left|\bar{w}_{N-1}\right| .
\end{aligned}
$$

For the other nodes with $i=2, \ldots, N-1$ the bounds on the gain are given by

$$
\begin{aligned}
\left|\bar{w}_{i}\right| & =\frac{\alpha_{i, i-1}\left|\bar{w}_{i-1}\right|}{\sqrt{\begin{array}{c}
\left(\alpha_{i, i}-\alpha_{i, i+1} \beta_{i+1, i}(\omega, \varphi) \cos \left(\varphi_{i+1}-\varphi_{i}\right)\right)^{2} \\
+\left(\omega-\alpha_{i, i+1} \beta_{i+1, i}(\omega, \varphi) \sin \left(\varphi_{i+1}-\varphi_{i}\right)^{2}\right.
\end{array}}} \\
& =\beta_{i, i-1}(\omega, \varphi)\left|\bar{w}_{i-1}\right|<(1-\gamma)\left|\bar{w}_{i-1}\right| .
\end{aligned}
$$

The relationship for the source node is

$$
\left|\bar{w}_{1}\right|<\frac{1}{\sqrt{\begin{array}{c}
\left(\alpha_{1,1}-\alpha_{1,2} \beta_{2,1}(\omega, \varphi) \cos \left(\varphi_{2}-\varphi_{1}\right)\right)^{2} \\
+\left(\omega-\alpha_{1,2} \beta_{2,1}(\omega, \varphi) \sin \left(\varphi_{2}-\varphi_{1}\right)\right)^{2}
\end{array}}} u .
$$

By inspection, it can easily be noticed that with $\omega=0$ and all $\varphi_{i+1}-\varphi_{i}=0$, we get that $\beta_{i, j}(0,0)$ correspond to $\beta_{i, j}$ 
from the constant input case such that the results from the constant input are recovered, and are in fact generalized here. Notice also that the constant input case $\beta_{i, j}(0,0)$ is always an upper bound for the sinusoidal case.

If we consider the general dynamics of subsystem $i$ described by (8) with sinusoidal input and neighbors in the set $\left\{j_{1}, \ldots, j_{l}\right\}$ one obtains for the steady state magnitude that

$$
\begin{aligned}
\begin{array}{r}
\left(\alpha_{i, j_{1}}\left|\bar{w}_{j_{1}}\right|+\sum_{j=j_{2}}^{j_{l}} \alpha_{i, j}\left|\bar{w}_{j}\right| \cos \left(\varphi_{j}-\varphi_{j_{1}}\right)\right)^{2} \\
+\left(\sum_{j=j_{2}}^{j_{l}} \alpha_{i, j}\left|\bar{w}_{j}\right| \sin \left(\varphi_{j}-\varphi_{j_{1}}\right)\right)^{2} \\
\sqrt{\alpha_{i i}^{2}+\omega^{2}}
\end{array} \\
\leq \sum_{j=j_{1}} \frac{\alpha_{i, j}}{\alpha_{i, i}}\left|\bar{w}_{j}\right| .
\end{aligned}
$$

We can see that the major difference to the case with constant input is that one cannot express the steady state magnitude as a linear combination of the neighboring steady state magnitudes, but that it can be bounded by the same linear combination obtained in the constant input case. Hence, the steady state magnitude in the sinusoidal case is always bounded by the steady state of the constant input case. Therefore, we can state the following corollary, omitting the proof.

Corollary 1: Given the system dynamics (11), and a sinusoidal input $u(t)=\sin (\omega t)$ at a designated source node, the steady state magnitude of a node $v$ with distance $d$ from the source node is upper bounded by an SSCC as defined in (12) of the steady state magnitudes of the unique $(d-1)$-nodes of node $v$.

\section{NUMERICAL EXAMPLE}

In this subsection, we will illustrate the obtained results in a numerical example.

We consider the following system dynamics for the $i$ th subsystem

$$
\begin{aligned}
& \dot{x}_{1, i}=x_{2, i} \\
& \dot{x}_{2, i}=-k_{0, i} x_{1, i}-b_{0, i} x_{2, i}+\sum_{j \in \mathcal{N}_{i}}\left(k_{1, i} x_{1, j}+b_{1, i} x_{2, j}\right),
\end{aligned}
$$

and we assume that the interconnection topology is given by the line graph shown in Figure 2 . We consider $N=10$ subsystems, and the parameters $k_{0, i}$ and $b_{0, i}$ are chosen from the set $\{1,2,3\}$ while the parameters $k_{1, i}$ and $b_{1, i}$ are chosen randomly from the set $\{0.01,0.02,0.03,0.04,0.05\}$. The parameters are chosen this way to ensure that a $\mathcal{A}$ exists such that $-\mathcal{A}$ is an M-matrix. The system dynamics can be imagined to represent a platoon of vehicles where the velocity of the $i$ th vehicle is influenced by the position and the velocity of the preceding and the following vehicle, e.g. through a control law, or because of an actual physical connection.

Given the system matrix $A$, we first construct $\tilde{\mathcal{A}}$ where we choose $Q_{i}$ to be the identity matrix of size two. Then we determine a diagonal matrix $D$ such that $\mathcal{A}=D^{-1} \tilde{\mathcal{A}} D$ is row diagonally dominant. The resulting reduced system dynamics are described by (11) where

$\mathcal{A}=\left[\begin{array}{cccccccccc}-0.96 & 0.86 & 0 & 0 & 0 & 0 & 0 & 0 & 0 & 0 \\ 0.09 & -0.31 & 0.19 & 0 & 0 & 0 & 0 & 0 & 0 & 0 \\ 0 & 0.5 & -0.57 & 0.01 & 0 & 0 & 0 & 0 & 0 & 0 \\ 0 & 0 & 0.48 & -1.14 & 0.05 & 0 & 0 & 0 & 0 & 0 \\ 0 & 0 & 0 & 0.07 & -0.97 & 0.22 & 0 & 0 & 0 & 0 \\ 0 & 0 & 0 & 0 & 0.14 & -0.58 & 0.15 & 0 & 0 & 0 \\ 0 & 0 & 0 & 0 & 0 & 0.34 & -0.87 & 0.09 & 0 & 0 \\ 0 & 0 & 0 & 0 & 0 & 0 & 0.1 & -1.1 & 0.26 & 0 \\ 0 & 0 & 0 & 0 & 0 & 0 & 0 & 0.06 & -0.44 & 0.18 \\ 0 & 0 & 0 & 0 & 0 & 0 & 0 & 0 & 0.44 & -0.83\end{array}\right]$

and we choose $b=[1,0, \ldots, 0]^{T}$.

In this case $\gamma=0.1021$. Therefore we expect the gain between hops from subsystem to subsystem to be at most $(1-$ $\gamma)=0.8979$. The response of the system to the constant input signal $u(t)=1$ is shown in Figure 3 . The steady state values $\bar{w}_{i}$ of the subsystems is shown in Figure 4 . The spatial decay can be clearly observed. The gain between hops $\left(\frac{\bar{w}_{i}}{\bar{w}_{i-1}}\right)$ lies between 0.0745 and 0.8808 and is shown in Figure 5. It can be seen that the upper bound $(1-\gamma)$ per hop is clearly conservative in general but in one case it is very close to the actual gain. Next, we apply a sinusoidal input $u(t)=\sin (\omega t)$ to the system. The system response for $\omega=1$ is shown in Figure 6 and one can easily see 1) the spatial decay between nodes, and 2) the phase shift between hops. To illustrate the spatial decay, the steady state magnitudes are also added to Figure 4 . The gain between hops, now described by $\frac{\left|\bar{w}_{i}\right|}{\left|\bar{w}_{i-1}\right|}$, is between 0.0484 and 0.4316. All the values, in addition to the values of the gain for $\omega=0.5$ and $\omega=2$, are shown in Figure 5 . Clearly, the constant input case is an upper bound for the sinusoidal case, as expected from the general analysis. Also it can be seen that while the bound is close in some cases, it is quite conservative in others and it becomes more and more conservative for increasing $\omega$. This is also expected. However, it seems that at least for relatively small values of $\omega$, the constant input case can be considered to be a good approximation as a bound for the sinusoidal case and offers easier computations.

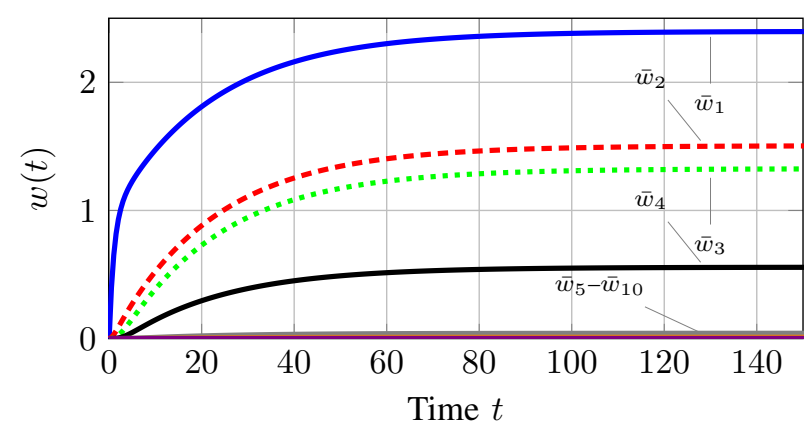

Fig. 3: Response to $u(t)=1$ for line-graph 


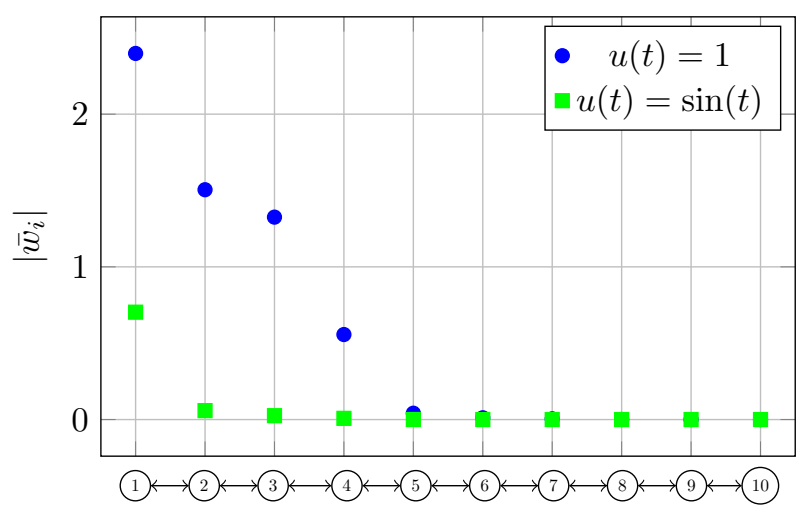

Fig. 4: Steady state values showing spatial decay for line graph

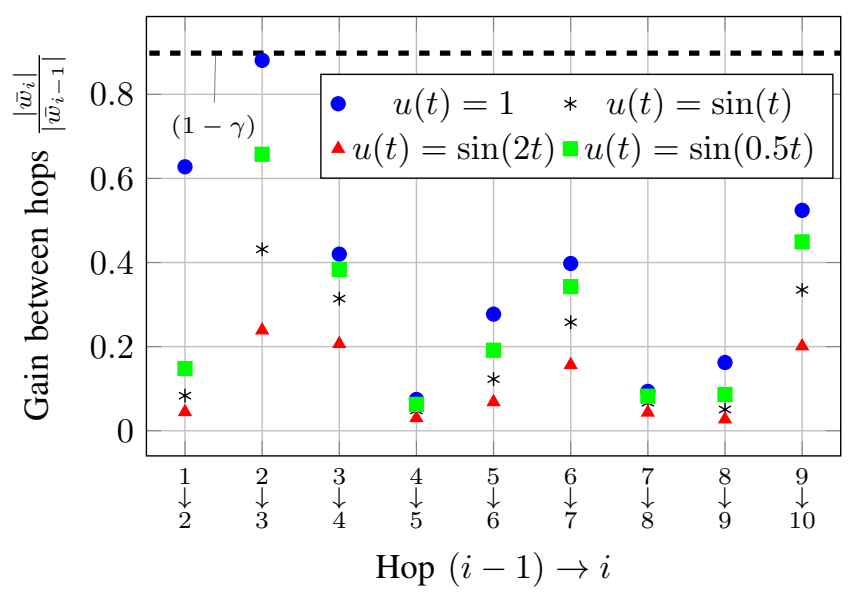

Fig. 5: Actual gain between hops with upper bound $(1-\gamma)=0.8979$.

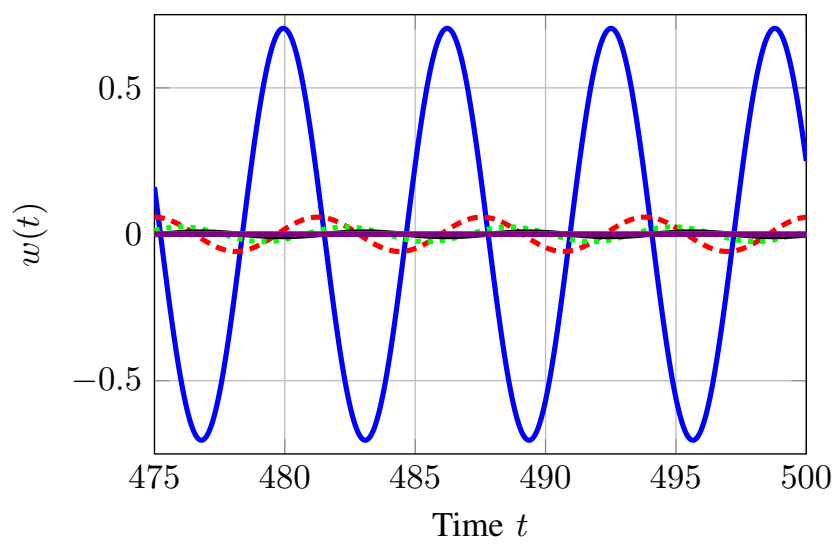

Fig. 6: Response to $u(t)=\sin (t)$ for line-graph

\section{CONCLUSIONS}

In this paper we have investigated the propagation of an input signal through a dynamical system. In order to achieve this the individual subsystems are represented by a scalar approximation such that the overall system is in the form of a vector Lyapunov function. We then assume that the new system dynamics matrix satisfies an M-matrix condition. For two special cases, a constant input and a sinusoidal input, analytical analysis of the steady state values has been carried out to see what the gain is between hops from subsystem to subsystem. The main result is that the steady state value of a node with a specific distance $d$ to the source node can be expressed as a so-called steady state conic combination of the steady state values of nodes with distance $(d-1)$ to the source node. For special cases like line-graphs or trees, the gain between hops can be bounded by a parameter $\gamma$ that is in direct relation to the entries of the system dynamics matrix. Also it has been seen that the constant input case can serve as an upper bound to the case with sinusoidal input. The results are validated and illustrated using numerical simulations. Future work will include transient analysis and changes in system parameters.

\section{ACKNOWLEDGMENTS}

The work of Frederik Deroo is supported by the TUM Munich School of Engineering. The work of Brian D. O. Anderson is supported by NICTA, which is funded by the Australian Government as represented by the Department of Broadband, Communications and the Digital Economy and the Australian Research Council through the ICT Centre of Excellence program, and the Australian Research Councils Discovery Project DP-110100538 and DP-130103610.

\section{REFERENCES}

[1] F. Deroo, M. Ulbrich, B. Anderson, and S. Hirche, "Accelerated iterative distributed controller synthesis with a barzilai-borwein step size," in 51st IEEE Conference on Decision and Control (CDC), 2012, pp. 4864-4870.

[2] G. Meurant, "A review on the inverse of symmetric tridiagonal and block tridiagonal matrices," SIAM Journal on Matrix Analysis and Applications, vol. 13, no. 3, pp. 707-728, 1992.

[3] T. Fujimoto, C. Herrero, and A. Villar, "A sensitivity analysis for linear systems involving m-matrices and its application to the leontif model," Linear Algebra and its Applications, vol. 64, pp. 85-91, 1985.

[4] F. Lin, M. Fardad, and M. R. Jovanovic, "Performance of leaderfollower networks in directed trees and lattices," in 51st IEEE Conference on Decision and Control (CDC), 2012, pp. 734-739.

[5] A. Rantzer, "Distributed control of positive systems," in 50th IEEE Conference on Decision and Control (CDC), 2011, pp. 6608-6611.

[6] K. Cai, B. D. O. Anderson, and C. Yu, "Local average consensus in distributed measurement of spatial-temporal varying parameters: 1D case," in 52nd IEEE Conference on Decision and Control (CDC), 2013, pp. 2139-2144.

[7] D. D. Siljak, Decentralized control of complex systems. Courier Dover Publications, 2011.

[8] A. Berman and R. J. Plemmons, Nonnegative matrices in the Mathematical Sciences. SIAM, 1979.

[9] D. D. Siljak, Large-Scale Dynamic Systems: Stability and Structure. North-Holland, 1978.

[10] M. Ikeda and D. D. Šiljak, "On decentrally stabilizable large-scale systems," Automatica, vol. 16, no. 3, pp. 331-334, 1980.

[11] J. L. Gross and J. Yellen, Handbook of graph theory. CRC press, 2004. 